Review Article

\section{Leakage after sleeve gastrectomy: Endoscopic stenting VS surgical intervention}

\author{
Mohamed Salah Abdelhamid ${ }^{1 *}$, Ahmed Zaki Gharib², \\ Mohammed Abdelaal Mohammed ${ }^{2}$ and Tarek Mahmoud \\ lbraheem El Gindy²
}

${ }^{1}$ Department of General Surgery, Faculty of Medicine, Beni- Suef University, Egypt

${ }^{2}$ Department of General Surgery, Faculty of Medicine, October 6 University, Egypt

\section{Abstract}

Background: Laparoscopic sleeve gastrectomy (LSG) is becoming more popular in the treatment of obesity. LSG is safe with a low morbidity. The complications rarely result in morbidity and even mortality. Leaks are the major complication associated with LSG with a reported prevalence between $1.9 \%$ and $2.4 \%$.

Objective: To compare surgical intervention and endoscopic stenting for treatment of gastric leakage after sleeve gastrectomy.

Patients and method: Our study included 30 patients presented with post sleeve leaks discovered by routine postoperative imaging or during the follow up period. Patients were recruited from October 6th university hospital during the period from August 2017 to August 2019. Patients were divided to the following groups: 1) Endoscopy group: This included 15 patients with post sleeve leakage undergoing endoscopic stent insertion. 2) Surgery group: which included 15 patients with post sleeve leak age undergoing surgical management. This division was random.

Results: Our study showed that Endoscopic stenting for management of post sleeve gastrectomy leakage is an effective method with lower morbidity and shorter post-operative hospital stay than surgical management. Some patients may be good candidates for early surgical intervention in type 1 leakage if managed early before dissemination of leakage and before tissues become friable. Complications of stents include stent migration $(26 \%)$, stent related ulcer $(13 \%)$ and stricture $(13 \%)$. while the surgical intervention carries more complications (DVT, chest infection, wound infection and stricture) and longer postoperative hospital stay.

Conclusion: endoscopic management of post-sleeve gastrectomy leakage with stenting is recommended because it successfully manages the leaks and avoids invasive procedures with less risk, with shorter hospital stay and early return of function.

\section{More Information}

*Address for Correspondence:

Mohamed Salah Abdelhamid, MD, Department of General Surgery, Faculty of Medicine, Beni- Suef University, Egypt, Tel: 00201007147991 ; Email: mohamedsalah_2000@hotmail.com

Submitted: 09 July 2020

Approved: 23 July 2020

Published: 24 July 2020

How to cite this article: Abdelhamid MS, Gharib AZ, Mohammed MA, El Gindy TMI. Leakage after sleeve gastrectomy: Endoscopic stenting VS surgical intervention. Arch Surg Clin Res. 2020; 4: 029-034.

DOI: 10.29328/journal.ascr.1001049

Copyright: @ 2020 Abdelhamid MS, et al. This is an open access article distributed under the Creative Commons Attribution License, which permits unrestricted use, distribution, and reproduction in any medium, provided the original work is properly cited.

Keywords: Sleeve gastrectomy; Stenting; Leakage

\section{(D) Check for updates}

(1) OPEN ACCESS

\section{Introduction}

Laparoscopic sleeve gastrectomy (LSG) is getting more popularity for the treatment of morbid obesity. LSG is a safe procedure with a low complication rate. The complications encountered nevertheless can result in morbidity and even mortality. The most significant complications are staple-line bleeding, stricture, and staple-line leak [1].

Leaks are the major complication associated with LSG with a reported prevalence between $1.9 \%$ and $2.4 \%$. Most leaks occur at the proximal part of the staple line, at the oesophagogastric junction [2].
Leaks can be classified based either on the time of onset (early, intermediate and late leaks), clinical presentation, site of leak, radiological appearance, or mixed factors. By clinical relevance and extent of dissemination, they defined type I or subclinical leaks and Type II leaks [3].

Based on both clinical and radiological findings, type A are microperforations without clinical or radiographic evidence of leak, while type B are leaks detected by radiological studies but without any clinical finding, and finally, type $\mathrm{C}$ are leaks presenting with both radiological and clinical evidence [4].

Diagnosis of a gastric leak can be difficult, as the 
presentation can vary from asymptomatic to severe septic shock. Usual symptoms may be of the septic nature: fever, tachycardia, tachypnea, leukocytosis, abdominal pain, and peritonitis, tachycardia $>120$ beats per minute (bpm) may be the most diagnostic sign of a gastric leak. 1 Burgos, et al. reported 7 leaks in 214 patients (3.3\%), of which 5 patients presented abdominal pain, fever, tachycardia, tachypnea and increased laboratory signs of infection. They observed that tachycardia is an initial sign of early leak [4].

Debates still exist on which diagnostic modality is the most sensitive and specific concerning the diagnosis of a post sleeve gastrectomy leakage, but all of them agree that early detection is associated with better outcome, and that a high index of suspicion is the cornerstone in the detection and diagnosis of leak [3].

The management of leak post sleeve gastrectomy imposes a lot of controversies and difficulties in the adoption of a standard algorithm, classification for gastric leakage post sleeve may constitute the first step in the establishment of such an algorithm or protocol, based on 3 characteristics: Time of appearance (early, intermediate and late); Location (proximal, mid or distal gastric); Severity or magnitude (typeIand II) [3].

Treatment principles for leaks that occur after bariatric surgeryinclude medical management(intravenous antibiotics and nutritional support), drainage of extra digestive soiling and stopping the leakage. Only once adequate drainage of any leak- associated fluid collections has been performed closure can be considered [5].

Classic surgical management of leaks consists of early reintervention to close or patch the gastric or anastomotic defect. These surgeries are associated with high morbidity and mortality. Reinterventions such as gastrectomy, gastric bypass on a complicated sleeve, or fistulojejunal anastomosis might be associated with less morbidity when performed on a more chronic defect [6].

Endoscopic management of postsurgical leaks offers the advantage of being less aggressive than surgery and include techniques to: cover (or exclude) the defect; close the defect; or temporarily maintain the defect in an open configuration to provide for internal drainage of the fluid collection [7].

Endoscopic options to treat leakage include: partially covered metallic self-expandable stent (PCSES) or fully covered stents, clipping of the defect, over-the-scope clips system (OTSCs), endoscopic insertion of a pigtail, fibrin sealant and suturing devices [8].

Over the last decade, there has been increasing use of selfexpanding metal stents (SEMS) for the treatment of sleeve leaks. The objective of stenting has been to divert gastric contents from the fistula site and to bypass the distal stenotic portion if present. Casella and colleagues reported the use of endoscopic stents for sleeve leaks in three patients with $100 \%$ success [9].

Surgicaloptions for leak management include primary repair and bowel, gastric or omentalpatching.

Considering surgical re-exploration for bariatric leaks carries an increased morbidity $(15 \%-50 \%)$ and mortality $(2 \%-10 \%)[10]$.

More definitive surgical options include: conversion of the LSG to a regular Roux-Y gastric bypass (RYGB) anastomosis of the jejunal Roux limb to the fistula and total gastrectomy [11].

\section{Aim of the work}

To compare surgical intervention and endoscopic stenting for treatment of gastric leakage after sleeve gastrectomy.

\section{Patients and methods}

\section{Study design}

This study will be conducted at October 6 university hospital during the period from August 2017 to August 2019 and will include 30 patients presented with post sleeve leaks discovered by routine postoperative imaging or during the follow up period. The study will include early hemodynamically stable type I leakage cases who failed to respond to conservative medical treatment. 15 patients will undergo endoscopic stent insertion and 15 patient will undergo surgical intervention. All patients were consented to participate in the study. Ethical approval from October 6 university hospital Ethical Committees was obtained.

Patient inclusion criteria: the study will include Type I leakage Hemodynamically stable patients.

Exclusion criteria: Unstable cases presented with septic shock or peritonitis.

\section{Methods}

Pre-operative assessment of a leaking patient included the following: History taking (age, sex, BMI, date of operation, onset of symptoms, history of acute or chronic illness). clinical examination including vital signs and local abdominal examination. Routine preoperative investigations (CBC, liver function tests, kidney functions test and electrolytes).

Radiological investigations (gastrograffinswallow, pelviabdominal US and CT scan.

\section{Management}

1. Drainage of the extra digestive space is part of the initial treatment strategy for all cases.

2. Conservative management include: nutritional support, IV antibiotics and good monitoring. 
3. Surgical management include: direct suturing for closure of the defect or patching of the defect.

4. Endoscopic management: insertion of partially covered metallic self-expandable stent (PCSES) for 6-8 weeks with the possibility of reinsertion of another stent after this period if leakage is not controlled (possible complications include: stent migration- failure to control-mucosal hyperplasia and ulceration).

\section{Postoperative assessment}

Follow up after management of leakage: to compare surgical management (group A) with endoscopic management (group B) will include: Follow up till patient is discharged from hospital by: Vital signs (pulse, temperature), drains output, early postoperative complications, post-operative hospital stay, CBC and CT scan for rate of resolution.

After hospital discharge: weekly follow up till leakage is controlled by: Evaluation of leak output and rate of control (drains, CT scan) and development of complications.

After leakage control: monthly follow up for 6 months for: Long term complications (recurrence, stricture).

\section{Statistical analysis}

An Excel spreadsheet was established for the entry of data. We used validation checks on numerical variables and option-based data entry method for categorical variables to reduce potential errors. The analyses were carried with SPSS software (Statistical Package for the Social Sciences, version 24 , SSPS Inc, and Chicago, IL, USA). The normality of the data were assessed using Shapiro-Wilk Test. Numerical data were described as mean \pm SD if normally distributed; or median and interquartile range [IQR] if not normally distributed. Frequency tables with percentages were used for categorical variables. Independent Student t-test and paired t-test were used to compare parametric quantitative variables; while Mann-Whitney tests and Wilcoxon matched pairs test were used to compare non-parametric quantitative variables. Chi-square test or McNemar-Bowker tests were used to analyze categorical variables. Multilinear logistic regression was undertaken to assess the predictors of mortality. A $p$-value $<0.05$ is considered statistically significant.

Prior to start we got approval from the ethical committee in our faculty with a written informed consent from every patient.

Table 1 shows that there was no statistically significant association between type of management and age $(p=0.88)$, BMI $(p=0.14)$, gender $(p=0.72)$, and comorbidities $(p=0.47)$.

Table 2 shows that there was no statistically significant association between type of management and leak site $(p=0.48)$ and Interval between surgery and leak ( $p=0.147)$.
Table 3 shows that there was no statistically significant association between type of management and initial treatment ( $p=0.47)$.

Table 4 shows that there was no statistically significant association between type of management and outcomes ( $p=0.51)$. In contrary, there was statistically significant association between type of management and interval between closure and leak control $(p<0.001)$ and hospital stay $(p=0.013)$. Patients underwent endoscopic management showed shorter hospital stay. The interval means that the patient became stable and not in need of more hospitalization.

\section{Discussion}

Laparoscopic sleeve gastrectomy (LSG) is emerging to be one of the commonly performed bariatric procedures worldwide for patients with different degrees of obesity. This restrictive procedure has several advantages. It is technically simpler to perform without the need of an anastomosis. It induces a reduction in ghrelin causing appetite suppression, which adds to the effect of restriction. It has been reported to have a lower morbidity and mortality rate in comparison to Roux-en-Y gastric bypass or biliopancreatic diversion with or without duodenal switch. It can be performed concomitantly with other procedures [12].

Table 1: The association between type of management and demographic characteristics of the included patients.

\begin{tabular}{|c|c|c|c|}
\hline Variables & $\begin{array}{l}\text { Endoscopy Group } \\
(n=15)\end{array}$ & $\begin{array}{l}\text { Surgery Group } \\
(n=15)\end{array}$ & $p$ - value \\
\hline \multicolumn{4}{|l|}{ Age in years } \\
\hline - Mean \pm SD & $34.93 \pm 7.7$ & $34.6 \pm 6.9$ & 0.88 \\
\hline - Median Range) & $35(22-44)$ & $33(24-46)$ & \\
\hline \multicolumn{4}{|l|}{$\mathrm{BMI}$ in $\mathrm{Kg} / \mathrm{m}^{2}$} \\
\hline - Mean \pm SD & $44.13 \pm 6.7$ & $43.53 \pm 6.6$ & 0.14 \\
\hline - Median (Range) & $44(23-69)$ & $43(32-57)$ & \\
\hline \multicolumn{4}{|l|}{ Gender, No (\%) } \\
\hline - Male & $6(40 \%)$ & $7(46.7 \%)$ & 0.71 \\
\hline - Female & $9(60 \%)$ & $8(53.3 \%)$ & \\
\hline \multicolumn{4}{|c|}{ Comorbidities, No (\%) } \\
\hline - DM & $3(20 \%)$ & $3(20 \%)$ & \\
\hline - HTN & $3(20 \%)$ & $1(6.7 \%)$ & 0.47 \\
\hline - HTN and DM & $4(26.7 \%)$ & $3(20 \%)$ & \\
\hline - Osteoarthritis & 0 & $2(13.3 \%)$ & \\
\hline
\end{tabular}

\begin{tabular}{|l|l|l|l|}
\hline Table 2: The characteristics of the leak. \\
\hline Variables & $\begin{array}{l}\text { Endoscopy Group } \\
(\boldsymbol{n}=15)\end{array}$ & $\begin{array}{l}\text { Surgery Group } \\
(\boldsymbol{n}=15)\end{array}$ & $p$ - value \\
\hline Leak site, No (\%) & & $11(73.3 \%)$ & \\
\hline - GE junction & $12(80 \%)$ & $1(6.7 \%)$ & 0.48 \\
\hline - Distal & & $3(20 \%)$ & \\
\hline - Mid-sleeve & $3(20 \%)$ & & \\
\hline
\end{tabular}

Table 3: Initial management of the leak of the included patients in both groups.

\begin{tabular}{|l|l|l|l|}
\hline Variables & $\begin{array}{l}\text { Endoscopy Group } \\
(\boldsymbol{n}=15)\end{array}$ & $\begin{array}{l}\text { Surgery Group } \\
(\boldsymbol{n}=15)\end{array}$ & $\boldsymbol{p}$ - value \\
\hline Initial treatment, No (\%) & & & \\
\hline - Fluids, ATB, NPO & $6(40 \%)$ & $5(33.3 \%)$ & 0.149 \\
\hline - Fluids, ATB, NPO, pigtail & $9(60 \%)$ & $10(66.7 \%)$ & \\
\hline
\end{tabular}


Table 4: Outcomes of the leak of the included patients in both groups.

\begin{tabular}{|c|c|c|c|c|c|c|c|c|}
\hline \multicolumn{6}{|c|}{ Variables } & Endoscopy Group $(n=15)$ & Surgery Group ( $n=15$ ) & $p$ - value \\
\hline \multicolumn{9}{|c|}{ Outcomes, No (\%) } \\
\hline- & \multicolumn{5}{|l|}{ Conversion to Roux en $\mathrm{Y}$} & $2(13.3 \%)$ & $1(6.7 \%)$ & \\
\hline- & \multicolumn{5}{|l|}{ Leak control } & $13(86.7 \%)$ & $13(86.7 \%)$ & 0.51 \\
\hline- & \multicolumn{5}{|l|}{ Mortality } & 0 & $1(6.7 \%)$ & \\
\hline \multicolumn{9}{|c|}{ Interval between closure days } \\
\hline - & Mean \pm SD & \multirow[t]{2}{*}{ and } & \multirow[t]{2}{*}{ leak } & \multirow[t]{2}{*}{ control } & \multirow[t]{2}{*}{ In } & $3.36 \pm 5.4$ & $5 \pm 4.1$ & \\
\hline - & Median (Range) & & & & & $35(28-42)$ & $3(2-16)$ & $<0.001$ \\
\hline \multicolumn{9}{|c|}{ Hospital stay in days } \\
\hline- & \multicolumn{5}{|l|}{ Mean \pm SD } & $8.7 \pm 4.1$ & $12.8 \pm 5.6$ & 0.013 \\
\hline- & \multicolumn{5}{|l|}{ Median (Range) } & $7(4-18)$ & $10(7-25)$ & \\
\hline
\end{tabular}

*Data are presented as mean $\pm \mathrm{SD}$, median (Range), or number (\%).

LSG can be associated with three significant complications, which include staple line gastric bleeding, staple line gastric leaks and gastric strictures. Of these, a gastric leak after sleeve gastrectomy is associated with significant and prolonged morbidity, remaining one of the most feared complications. Gastric leak can present as peritonitis, abscesses, cutaneous or other fistulas, sepsis, organ failure and even death [13].

The treatment for leaks after sleeve gastrectomy varies and depends upon the extent of disruption, the extent of abdominal contamination, and the site of leak (proximal versus distal). Endoscopy is an excellent first line tool and may be simultaneously diagnostic and therapeutic. Endoscopic stents were initially designed as a tool of palliation for obstructing esophageal, gastric, and colorectal cancer. Over the recent years, a growing body of evidence has shown that stent placement for gastro-jejunal leaks is a safe therapeutic option [14].

Nevertheless, there is a scarcity in the published literature regarding the efficacy and safety of endoscopic stenting for management of leakage after LSG. Therefore, we conducted the present study in order to compare surgical intervention and endoscopic stenting for treatment of gastric leakage after LSG.

In the present study, the mean age of the included patients was around 35 years old and the majority of the patients were females. In addition, more than two- third of the patients had one or more comorbidities this was in agreement with, Juza and colleagues [15].

Leaks can be classified based either on the time of onset, clinical presentation, site of leak, radiological appearance, or mixed factors. Csendes, et al [16]. defined early, intermediate and late leaks as those appearing 1 to 4,5 to 9 and 10 or more days following surgery respectively The most frequent site of leak, in a vast majority of patients with $\mathrm{SG}$, is proximal, near the gastroesophagal (GE) junction [14].

In line with these findings, Sakran and colleagues [17] performed a retrospective analysis by querying 2,834 patients who underwent LSG, $44(1.5 \%)$ with gastric leaks were identified. Of these 44 patients, 30 (68\%) were women. The patients had a mean age of 41.5 years Leaks were diagnosed at a median of 7 days postoperatively: early (0-2 days) in nine cases (20\%), intermediately (3-14 days) in 32 cases (73\%), and late (>14 days) in three cases (7\%). In 33 of the patients $(75 \%)$, the leak site was found in the upper sleeve near the gastroesophageal junction.

Similarly, Vix and colleagues [18] aimed to report the leak rate and its management in 378 LSGs. The overall leak rate was $9 / 378(2.38 \%)$. The leaks were mostly at GE junction and intermediate.

Regarding the primary outcomes of the present study, the endoscopic stents achieved leak closure in 13 (86.7\%) patients and only 2 patients required conversion to Roux en $Y$. The interval between closure of leak and leak control was $3.36 \pm 5.4$ days. The average hospital stay was $8.7 \pm 4.1$ days. The analysis showed that there was no statistically significant association between type of management and outcomes $(p=0.51)$. In contrary, there was statistically significant association between type of management and interval between closure and leak control $(p<0.001)$ and hospital stay $(p=0.013)$. Patients underwent endoscopic management showed shorter interval till leak control and hospital stay.

In concordance with our findings, Puli and colleagues [19] performed a systematic review and meta-analysis to evaluate the success of self- expandable stents (SESs) in the treatment of bariatric surgery leaks. A total of 189 relevant articles were reviewed of which 7 studies (67 patients with leaks) met inclusion criteria. The pooled proportion of successful leak closures by using SESs was 87.77\% (95\% CI, 79.39\%94.19\%). The pooled proportion of successful endoscopic stent removal was $91.57 \%$ (95\% CI, 84.22\%-96.77\%).

Similarly, Murino and colleagues [20] evaluated the effectiveness of a endoscopic stents for management of postbariatric surgery leaks in a large cohort of patients. Data from patients with anastomotic leaks after bariatric surgery endoscopically treated with partially covered SEMS between January 2006 and December 2012 were retrospectively reviewed. The stenting policy was successful to close the leak in $88 \%$ of the patients.

Southwell and colleagues [21] described the experience 
of endoscopic management of post- LSG leaks. A total of 21 patients have received endotherapy for post-LSG leak management. Treatment included the deployment of primary SEMS across the leak site. A total of 20/21 (95\%) patients had resolved leaks following a mean of 75 days of treatment (median 47, range 9-187).

While, Garofalo and colleagues [22] included a total of 872 LSGs, overall, 10 of 872 patients (1.1\%) developed a gastric leak. Endoscopic fistula closure at the gastroesophageal junction was achieved in 10 of 11 cases (90\%) and the average time for closure was 9.9 (range: 4-24) weeks.

Moreover, Tsai and colleagues [23] evaluated the efficacy and complications of SEMS in the treatment of post-bariatric surgical leak. seven patients underwent covered SEMS placement for leak after bariatric surgery, including LSG $(n=6)$ Among six patients, one patient who received stent placement one year after leak diagnosis failed to achieve leak closure, and five patients with early stent placement achieved leak closure (83.3\%).

Eubanks and colleagues [24] presented the outcomes of large series to date treating staple line complications after bariatric surgery with endoscopic covered stents. A retrospective evaluation was performed of all patients treated for staple line complications after bariatric surgery at a single tertiary care bariatric center. 19 patients were treated with a total of 34 endoscopic silicone covered stents. Resolution of leak after stent treatment occurred in 16 of 19 patients (84\%).

Complications of stent insertion include early removal of the stent due to stent migration, bleeding, and obstruction due to kinking of the proximal portion of the stent. Stent migration is the main drawback of the technique requiring stent removal [25].

In the present study, the incidence of short-term complications in endoscopy group were, restenting due to failure of therapy (6.7\%), stent migration (26.7\%), and subphrenic abscess (6.7\%). In long-term follow-up, 2 patients (13.4\%) developed stent-related ulcer and a similar number of patients developed stricture.

In agreement with our findings, Campo and colleagues [26] described single-institution experience in managing SG leaks with endoscopic stents. Data for all patients who underwent endoscopic stent placement for an SG leak Twenty-four patients with SG staple-line leaks treated with covered endoscopic stents were identified. Migration occurred in $22 \%$ of all stent placements.

Botaitis, et al. [27] support primary surgical repair of the defect and immediate surgical intervention with wash out drainage and primary repair for patients with early leaks due to the fact that the surrounding tissues are still healthy.
More definitive surgical options include: conversion of the LSG to a regular Roux-Y gastric bypass (RYGB), anastomosis of the jejunal Roux limb to the fistula and total gastrectomy. Gomes suggested that converting a sleeve to a RYGB leads to decompression of the high intragastric pressure within the sleeve to a low pressure system. Also a Roux limb allows for better drainage than a sleeve, which can have functional disorders or stenotic areas. Conversion of the LSG to a RYGB may not be advisable in the presence of significant peritonitis [11] studies have shown that early re-suturing within the first three days can result in successful closure versus resuturing leaks after the third day. Hence this is considered as a 'favorable' window period and attempt at early surgical closure of the defect may be performed when re-exploration is early and tissues are healthy. If possible re-sleeve of the fistula site by stapling can be done with suture reinforcement [12].

In our study 7 patients were managed by early surgical intervention with drainage and direct suturing for the leak site as the tissues weren't friable and the repair was successful.

In our study, In the surgery group, the incidence of shortterm complications was as the following in surgery group:: chest infection (6.7\%), DVT (6.7\%), wound infection (6.7\%), severe vomiting (6.7\%), and sub-phrenic abscess (6.7\%). In long-term follow-up, 2 patients (13.4\%) developed stricture.

Despite the overall complications rates were comparable between endoscopy and surgery arms, there were notable more severe complications in the surgery group. DVT, wound infection, and severe vomiting can have devastating consequences in postoperative period. For example, the autopsy studies document that $50 \%$ of all patients dying in hospital have DVT. Such findings may explain the favorable outcomes of endoscopic stenting in terms of hospital stay and time to return to normal function.

\section{Conclusion}

Endoscopic management of post-sleeve gastrectomy leakage with stenting is recommended because it successfully manages the leaks and avoids invasive procedures with less risk. , with shorter hospital stay and early return of function.

\section{References}

1. Dakwar A, Assalia A, Khamaysi I, Kluger Y, Mahajna A. Late complication of laparoscopic sleeve gastrectomy. Case Rep Gastrointest Med. 2013; 136153.

PubMed: https://pubmed.ncbi.nlm.nih.gov/23662218/

2. Zellmer JD, Mathiason MA, Kallies KJ, Kothari SN. Is laparoscopic sleeve gastrectomy a lower risk bariatric procedure compared with laparoscopic Roux-en-Y gastric bypass? A meta- analysis. Am J Surg. 2014; 208: 903-910.

PubMed: https://pubmed.ncbi.nlm.nih.gov/25435298/

3. Rached AA, Basile M, El Masri H. Gastric leaks post sleeve gastrectomy: Review of its prevention and management, World $\mathrm{J}$ 
Gastroenterol. 2014; 20: 13904-13910.

PubMed: https://www.ncbi.nlm.nih.gov/pmc/articles/PMC4194572/

4. Burgos AM, Braghetto I, Csendes A, Maluenda F, Korn O, et al. Gastric leak after laparoscopic-sleeve gastrectomy for obesity. Obes Surg 2009; 19: 1672-1676.

PubMed: https://pubmed.ncbi.nlm.nih.gov/19506979

5. Eisendrath P, Deviere J. Major complications of bariatric surgery: endoscopes as first-line treatment. Nature review Gastroenterology \& Hepatology. 2015.

6. van de Vrande S, Himpens J, El Mourad H, Debaerdemaeker R, Leman G. Management of chronic proximal fistulas after sleeve gastrectomy by laparoscopic Roux-limb placement. Surg Obes Relat Dis. 2013; 9: 856-861.

PubMed: https://pubmed.ncbi.nlm.nih.gov/23433751/

7. Leenders BJ, Stronkhorst A, Smulders FJ, Nieuwenhuijzen GA, Gilissen LP. Removable and repositionable covered metal selfexpandable stents for leaks after upper gastrointestinal surgery: experiences in a tertiary referral hospital. Surg. Endosc. 2013; 27" 2751-2759.

PubMed: https://pubmed.ncbi.nlm.nih.gov/23436082/

8. Mercky P, Gonzalez J, Bonin EA, Emungania O, Brunet J, et al. Usefulness of over-the-scope clipping system for closing digestive fistulas. Dig Endosc. 2014; 27: 18-24.

PubMed: https://pubmed.ncbi.nlm.nih.gov/24720574/

9. Casella G, Soricelli E, Rizello M, et al. Nonsurgical treatment of staple line leaks after laparoscopic sleeve gastrectomy. Obes Surg. 2009; 19: 821-826. PubMed: https://www.ncbi.nlm.nih.gov/pubmed/19381737

10. Docimo S, Pryor AD. Management of Leaks with Endoluminal Stents. In: Chand B. (eds) Endoscopy in Obesity Management. Springer, Cham. 2018.

11. Gomes RM, Palaniappan R, Bhasker A, Naik S, Shah S. Management of Leaks After Sleeve Gastrectomy. In: Palanivelu P, Kumar S, Gomes R. (eds) Bariatric Surgical Practice Guide. Springer, Singapore. 2017.

12. Praveenraj $P$, Gomes $R$, Kumar $S$, et al. Management of gastric leaks after laparoscopic sleeve gastrectomy for morbid obesity: A tertiary care experience and design of a management algorithm. J Minimal Access Surg. 2016; 12: 342-349.

PubMed: https://www.ncbi.nlm.nih.gov/pmc/articles/PMC5022516/

13. Schulman AR, Thompson CC. Endoscopic Evaluation/Management of Bariatric Surgery Complications. Current Treatment Options in Gastroenterology. 2017; 15: 701-716.

14. Walsh C, Karmali S. Endoscopic management of bariatric complications: A review and update. World Journal of Gastrointestinal Endoscopy. 2017; 7: 518.

PubMed: https://www.ncbi.nlm.nih.gov/pmc/articles/PMC4436919/

15. Juza RM, Haluck RS, Pauli EM, et al. Gastric sleeve leak: A single institution's experience with early combined laparoendoscopic management. Surg Obes Related Dis. 2015; 11: 60-64.

16. Csendes A, Burdiles P, Burgos AM, Maluenda F, Diaz JC.
Conservative management of anastomotic leaks after 557 open gastric bypasses. Obes Surg. 2005 15: 1252-1256.

PubMed: https://pubmed.ncbi.nlm.nih.gov/16259881

17. Sakran N, Goitein D, Raziel A, Keidar A, Beglaibter N, et al. Gastric leaks after sleeve gastrectomy: A multicenter experience with 2,834 patients. Surg Endosc. 2013; 27: 240-245.

PubMed: https://pubmed.ncbi.nlm.nih.gov/22752283/

18. Vix M, Diana M, Marx L, Callari C, Wu HS, et al. Management of staple line leaks after sleeve gastrectomy in a consecutive series of 378 patients. Surg Laparosc Endosc Percutan Tech. 2015; 25: 89-93. PubMed: https://www.ncbi.nlm.nih.gov/pubmed/24752161

19. Puli SR, Spofford IS, Thompson CC. Use of self-expandable stents in the treatment of bariatric surgery leaks: A systematic review and meta- analysis. Gastrointest Endosc. 2012; 75: 287-293.

PubMed: https://pubmed.ncbi.nlm.nih.gov/22047699

20. Murino A, Arvanitakis M, Le Moine $O$, et al. Effectiveness of Endoscopic Management Using Self-Expandable Metal Stents in a Large Cohort of Patients with Post-bariatric Leaks. Obesity Surgery. 2015; 25: 1569-1576.

21. Southwell T, Lim TH, Ogra R. Endoscopic Therapy for Treatment of Staple Line Leaks Post-Laparoscopic Sleeve Gastrectomy (LSG): Experience from a Large Bariatric Surgery Centre in New Zealand. Obes Surg. 2016; 26: 1155-1162.

PubMed: https://pubmed.ncbi.nlm.nih.gov/26475027/

22. Garofalo F, Noreau-Nguyen M, Denis R, Atlas H, Garneau P, et al. Evolution of endoscopic treatment of sleeve gastrectomy leaks: from partially covered to long, fully covered stents. Surg Obes Relat Dis. 2017; 13: 925-932.

PubMed: https://pubmed.ncbi.nlm.nih.gov/28237561/

23. Tsai YN, Wang HP, Huang CK, Chang PC, Lin IC, et al. Endoluminal stenting for the management of leak following sleeve gastrectomy and loop duodenojejunal bypass with sleeve gastrectomy. Kaohsiung $\mathrm{J}$ Med Sci. 2018; 34: 43-48.

PubMed: https://pubmed.ncbi.nlm.nih.gov/29310815/

24. Eubanks S, Edwards CA, Fearing NM, Ramaswamy A, de la Torre RA, et al. Use of4 Endoscopic Stents to Treat Anastomotic Complications after Bariatric Surgery. J Am Coll Surg. 2008; 206: 935-938. PubMed: https://pubmed.ncbi.nlm.nih.gov/18471727/

25. Anastasiou J, Eubanks S, Edwards CA, Fearing NM, et al. Use of Endoscopic Stents to Treat Anastomotic Complications after Bariatric Surgery. J Am Coll Surg. 2008; 206: 935-938. PubMed: https://pubmed.ncbi.nlm.nih.gov/18471727/

26. Martin del Campo SE, Mikami DJ, Needleman BJ, Noria SF. Endoscopic stent placement for treatment of sleeve gastrectomy leak: a single institution experience with fully covered stents. Surg Obes Relat Dis. 2018; 14: 453- 461.

PubMed: https://pubmed.ncbi.nlm.nih.gov/29370996/

27. Botaitis S, Mitsala A, Perente S, Simopoulos C. Prevention and Management of Staple Line Leaks after Laparoscopic Sleeve Gastrectomy. J Anesth Surg. 2018; 5: 95-102. 